\title{
Chimney Technique with Nellix EndoVascular Aneurysm Sealing System in a Patient with Single Kidney and Juxtarenal Abdominal Aortic Aneurysm
}

Kaspars Kisis, MD ${ }^{1,5^{*}}$, Janis Savlovskis, MD²,4 Marcis Gedins, MD' ${ }^{1}$, Natalija Ezite, MD $^{2,5}$, Edgars Zellans, $\mathrm{MD}^{3}$, Dainis Krievins, $\mathrm{MD}, \mathrm{PhD}^{1,4}$

${ }^{1}$ Department of Vascular Surgery, Pauls Stradins Clinical University Hospital, Riga, Latvia

2 Department of Radiology, Pauls Stradins Clinical University Hospital, Riga, Latvia

${ }^{3}$ Department of Anaesthesiology, Pauls Stradins Clinical University Hospital, Riga, Latvia

4 University of Latvia, Riga, Latvia

${ }^{5}$ Riga Stradins University, Riga, Latvia

\begin{abstract}
We present a saccular asymptomatic juxtarenal abdominal aortic aneurysm in a 70-year-old male with a very short left renal artery supplying the only kidney. The case was successfully treated with the Nellix EndoVascular Aneurysm Sealing system combined with a chimney technique.
\end{abstract}

Copyright @ 2016 Science International Corp.

\section{Key Words}

Abdominal aortic aneurysm - Endovascular aortic aneurysm repair • Chimney technique • Single kidney • EVAS

\section{Introduction}

Endovascular aneurysm repair (EVAR) has become the method of choice for abdominal aortic aneurysm (AAA) repair, especially in patients deemed intermediate or high risk for open aneurysm repair [1].
The Nellix EndoVascular Aneurysm Sealing (EVAS) System (Endologix, Inc., Irvine, CA, USA) is a relatively new endoluminal device designed to treat infrarenal AAA by obliterating the aneurysm sac, thus eliminating the potential endoleak space while maintaining normal flow to the lower extremities. The endograft blood-flow lumens are supported with balloon-expandable endoframes surrounded by polymer-filled endobags, and do not require proximal and distal fixation $[2,3]$. The goal of the EVAS method is to treat AAAs while preserving side branches.

\section{Case Presentation}

A 70-year-old male presented with several years of abdominal and back pain associated with a pulsatile abdominal mass. Computed tomographyangiography (CTA) was performed, showing a $4.7 \times$ 2.7-cm pararenal saccular AAA and a very short 0.8$\mathrm{cm}$ left renal artery. Both common iliac arteries were non-aneurysmal (Figure 1). The patient's comorbid-

\footnotetext{
* Corresponding Author:

Kaspars Kisis

Department of Vascular Surgery

Pauls Stradins Clinical University Hospital

13 Pilsonu Street, Riga, LV-1002, Latvia

Tel.: +3 712955 4977; Fax: +3 716706 9946; E-Mail: kaspars08@gmail.com
} 


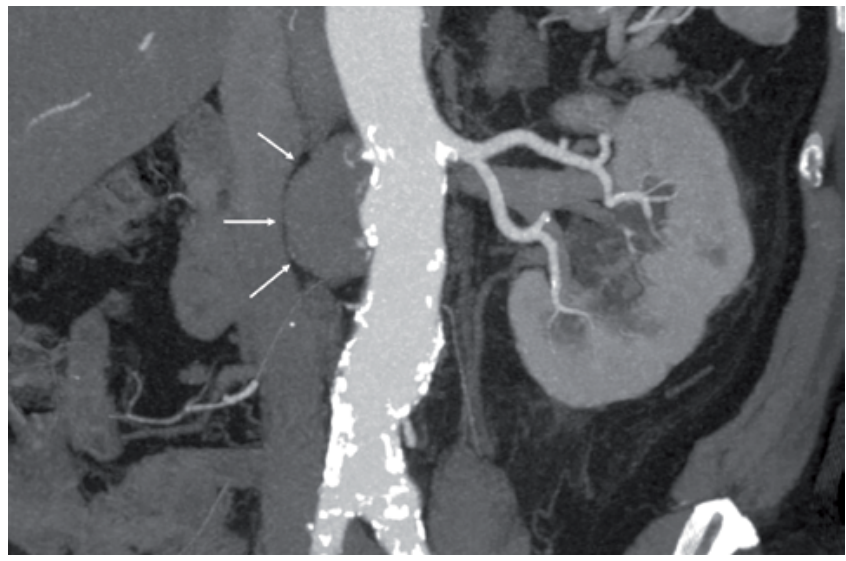

Figure 1. Pre-treatment computed tomography-angiography demonstrating a saccular $4.7 \times 2.7-\mathrm{cm}$ abdominal aortic aneurysm (arrows), the left solitary kidney, and a short $(0.8-\mathrm{cm})$ left renal artery.

ities included right kidney tumor and subsequent right adrenalectomy and nephrectomy three years previously, which may have caused the formation of the pseudoaneurysm in the aorta at the renal artery level. He also had hypertension and was previously a smoker. The patient had also experienced abdominal trauma 15 years previously caused by a falling tree. The other patient history was unremarkable.

The decision to offer an endovascular solution for this patient was based on the risk/benefit assessment of open surgery, which would involve suprarenal clamping and re-implantation of the single left renal artery. A chimney technique was selected to be combined with EVAS, because this method would preserve the single renal artery. The procedure was performed under spinal anesthesia in a hybrid operating theatre.

Percutaneous access was gained via the common femoral artery to introduce the Nellix system. We used two 160-mm Nellix stent endoframes. Devices were introduced into the abdominal aorta above the left renal artery to fully exclude the aneurysm. Renal artery access was gained via a left brachial artery cutdown using a $7 \mathrm{~F}$ catheter (Figure 2). A $7 \times 80-\mathrm{mm}$ percutaneous transluminal angioplasty (PTA) balloon was placed into the left renal artery to preserve a flow channel in this very short vessel during polymer injection into the endobags (Figure 3 ). Both endoframes were deployed and the endobags were filled with a polymer under pressure guidance. The EVAS

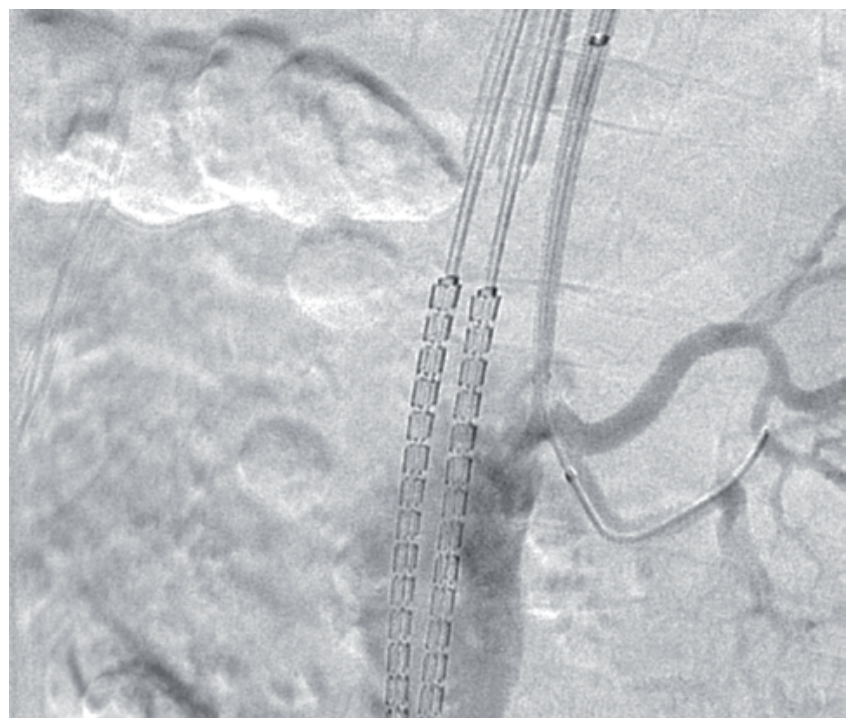

Figure 2. Chimney technique used to treat the abdominal aortic aneurysm involving the single left renal artery. Nellix devices were introduced into the aorta, and the left renal artery was cannulated.

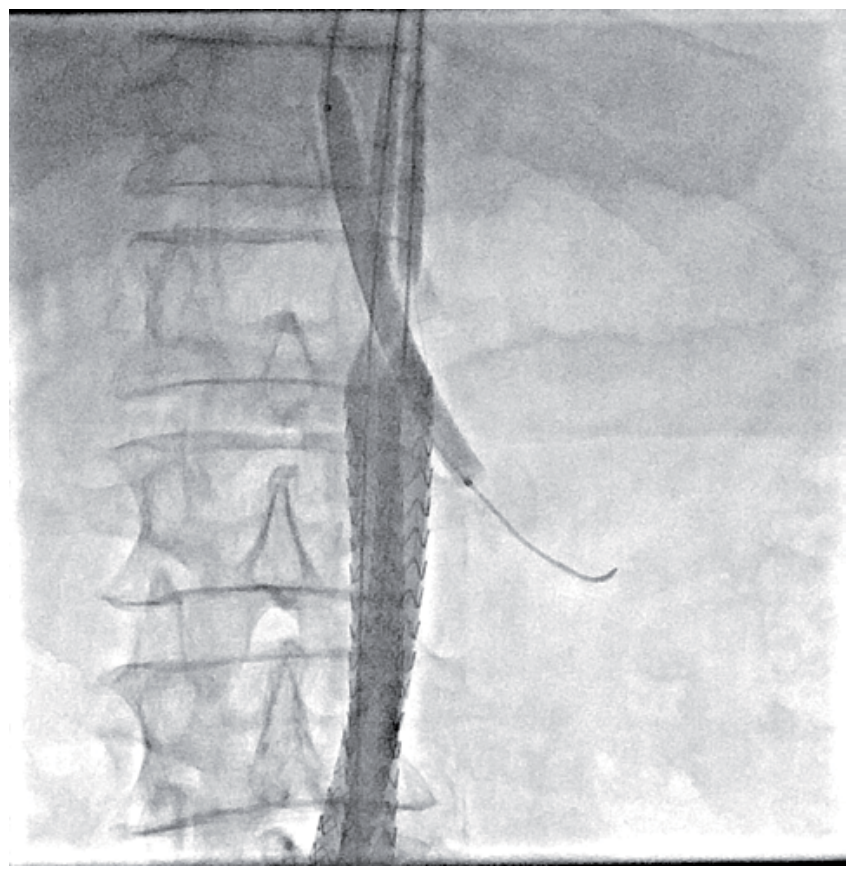

Figure 3. A percutaneous transluminal angioplasty balloon was expanded in the left renal artery at the time of deployment of the Nellix stents and polymer injection into the endobags.

procedure was carried out according to standard procedures [2, 3]. After polymer curing and exclusion of the aneurysm, the PTA balloon was removed and the flow channel between the endobags and the aortic 


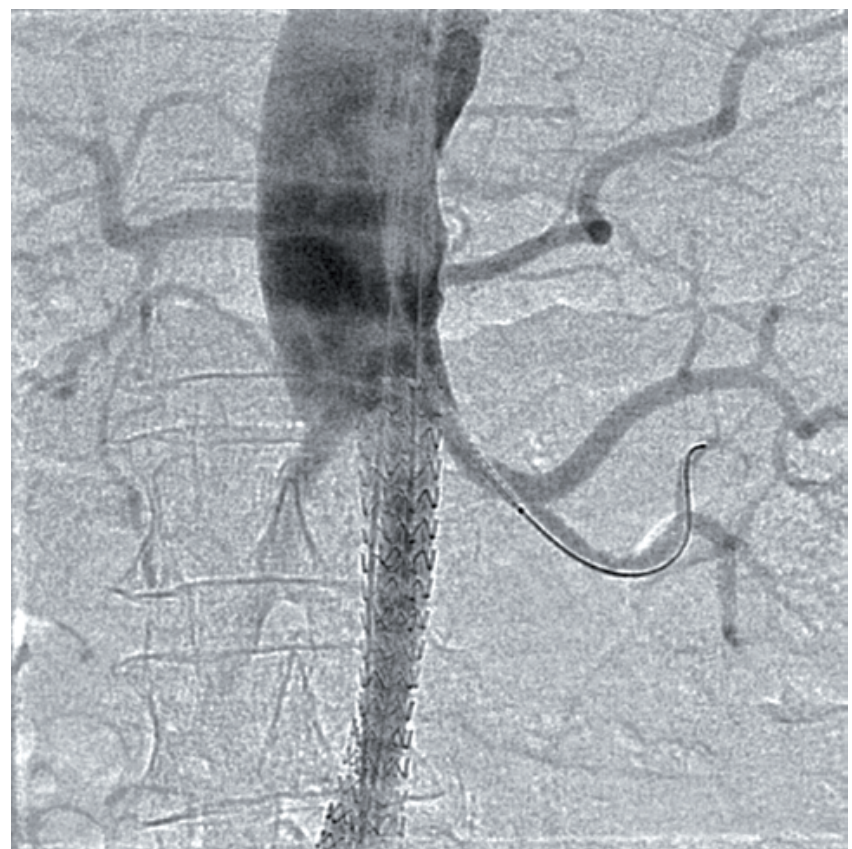

Figure 4. Stent implantation in the renal artery after endovascular aneurysm sealing to support a renal flow channel.

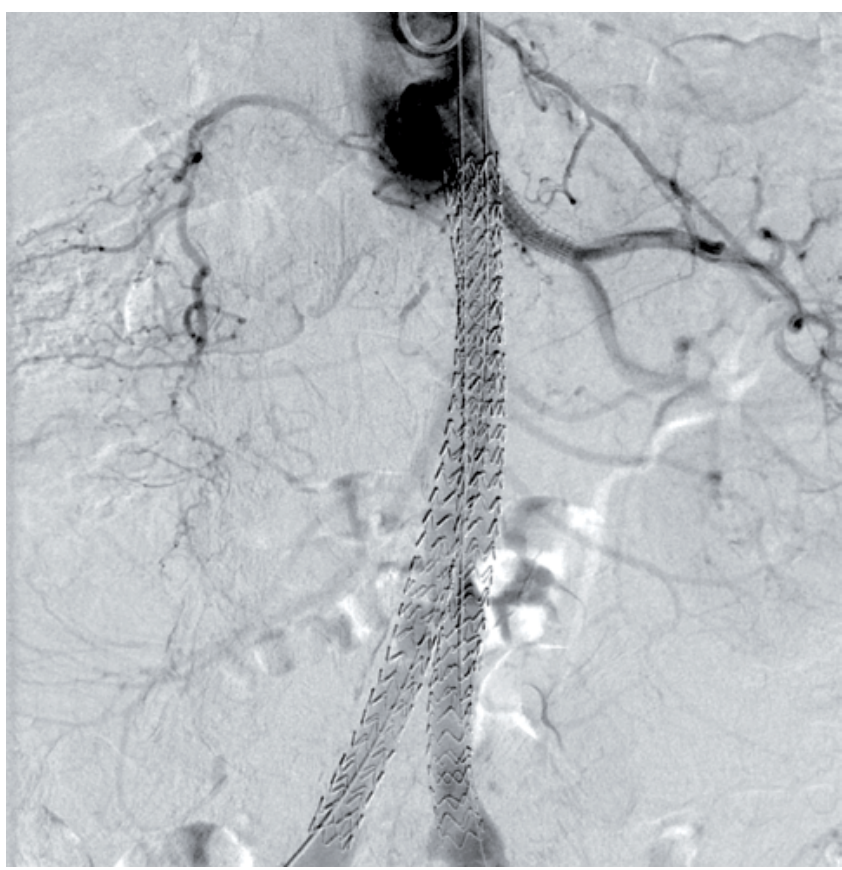

Figure 5. Post-procedural angiogram showing exclusion of the abdominal aortic aneurysm with a patent renal stent.

wall was supported by introducing an $8 \times 50-\mathrm{mm}$ Gore Viabahn stent graft (W. L. Gore \& Associates, Flagstaff, AZ, USA) (Figures 4 and 5). Access sites in

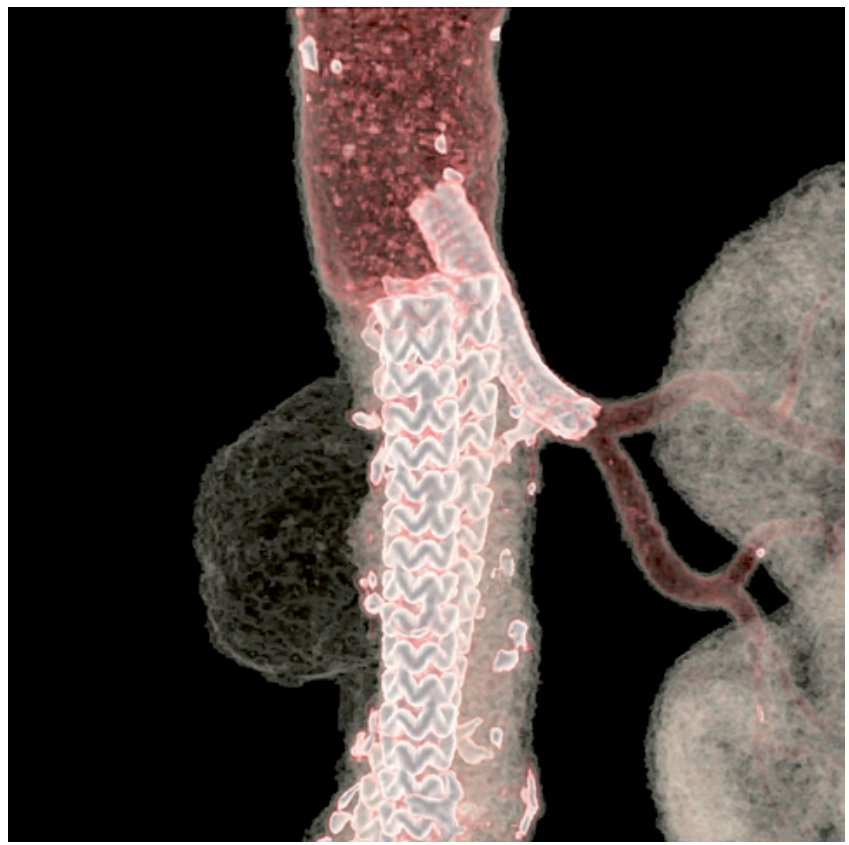

Figure 6. Six-month follow-up computed tomography-angiography demonstrating full abdominal aortic aneurysm exclusion and a patent renal artery.

the femoral arteries were closed using two Proglide vascular closure devices (Abbott Vascular, Santa Clara, (A, USA) and the brachial artery was closed by direct suture.

The patient had recovered fully and was discharged three days after the procedure with dual antiplatelet therapy for one year. No renal complications or deterioration of renal function were observed at the time of discharge or during follow-up at one or six months. One-month follow-up CTA showed fully patent renal arteries without branch artery thrombosis or renal infarctions. Six-month follow-up CTA scans showed full exclusion of the AAA with no change in diameter, and patent left renal artery (Figure 6). Renal function continues to be normal.

\section{Discussion}

Successful results have been reported for the chimney technique with EVAR stent grafts [4]. Nevertheless, Type-I endoleaks are frequently seen after treating patients with self-expanding stent grafts. The Nellix EVAS system can be used successfully for the treatment of challenging AAAs involving side 
branches [5]. This is a promising technology for performing chimney grafts with lower risk for Type- 1 endoleaks due to the polymer filling of the space around the stents.

Special difficulties were present in the case described here: the very short side branch posed a potential risk of intra-procedural stent migration from the side branch to the aorta. We present in this report a successful strategy for the creation of a flow channel at the time of EVAS and implantation of a supporting stent graft after the procedure.

This case shows that the Nellix EVAS system combined with a chimney technique can successfully treat

\section{References}

1. Brewster DC, Cronenwett JL, Hallett JW, Jr, Johnston KW, Krupski WC, Matsumura $J S$, et al. Guidelines for the treatment of abdominal aortic aneurysms. Report of a subcommittee of the Joint Council of the American Association for Vascular Surgery and Society for Vascular Surgery. J Vasc Surg. 2003;37:1106-1117. DOI: 10.1067/ mva.2003.363

2. Donayre $C D$, Zarins $C K$, Krievins DK, Holden $A$, Hill A, Calderas C, et al. Initial clinical experience with a sac-anchoring endoprosthesis for aortic aneurysm repair. J Vasc Surg. 2011;53:574-582. DOI: 10.1016/j. jvs.2010.09.009

3. Krievins DK, Holden A, Savlovskis J, Calderas $C$, Donayre CD, Moll FL, et al. EVAR us-
AAA and preserve the renal artery, opening new horizons for EVAS in the treatment of juxtarenal aneurysms.

\section{Conflict of Interest}

Dainis Krievins and Janis Savlovskis are paid consultants for the Endologix Nellix scientific medical advisory board.

\section{Comment on this Article or Ask a Question}

ing the nellix sac-sealing endoprosthesis: treatment of favourable and adverse anatomy. Eur J Vasc Endovasc Surg. 2011;42: 38-46. DOI: 10.1016/j.ejvs.2011.03.007

4. Ronchey S, Serrao E, Kasemi H, Pecoraro F, Fazzini S, Alberti V, et al. Endovascular treatment options for complex abdominal aortic aneurysms. J Vasc Interv Radiol. 2015;26:842-854. DOI: 10.1016/j. jvir.2015.02.021

5. Bockler $D$, Reijnen $M M$, Krievins $D$, Peters AS, Hayes P, De Vries JP. Use of Nellix EVAS system to treat post-EVAR complications and to treat challenging infrarenal necks. J Cardiovasc Surg. 2014;55:601-612. PMID: 25175947
Cite this article as: Kisis $\mathrm{K}$, Savlovskis J, Gedins M, Ezite N, Zellans E, Krievins D. Chimney Technique with Nellix EndoVascular Aneurysm Sealing System in a Patient with Single Kidney and Juxtarenal Abdominal Aortic Aneurysm. AORTA (Stamford). 2016;4(4):134-137. DOI: $\quad$ http://dx.doi.org/10.12945/j. aorta.2016.16.013 